\title{
A Study of the Prediction Requirements in Real-Time Control of Wave Energy Converters
}

\author{
Francesco Fusco and John V. Ringwood
}

\begin{abstract}
It is widely acknowledged that real-time control of wave energy converters (WECs) can benefit from prediction of the excitation force. The prediction requirements (how far ahead into the future do we need to predict?) and the achievable predictions (how far ahead can we predict?) are quantified when unconstrained reactive control is implemented. The fundamental properties of the floating system that influence the length of the required forecasting horizon, as well as the achievable prediction, are characterized. The possibility of manipulating the control, based on prior knowledge of the wave spectral distribution, is also proposed for the reduction of the prediction requirements, such that they are within the range of predictability offered by simple stochastic predictors. The proposed methodology is validated on real wave data and heaving buoys with different geometries.
\end{abstract}

Index Terms-Optimal control, predictive control, wave energy, wave forecasting.

\section{NOMENCLATURE}

$\begin{array}{ll}x(t), X(\omega) & \text { Fourier transform pair. } \\ v(t) & \text { Heaving position and velocity of floating body. } \\ f_{\mathrm{ex}}(t) & \text { Wave excitation force. } \\ f_{u}(t) & \text { Power takeoff force (control input). } \\ Z_{i}(\omega) & \text { Intrinsic impedance of floating system. } \\ Z_{u}(\omega) & \text { Impedance of power takeoff. } \\ B(\omega) & \text { Radiation resistance of floating system. } \\ \Delta \omega & \text { Bandwidth of } B(\omega) . \\ H_{\mathrm{opt}}(\omega) & \begin{array}{l}\text { Transfer function between excitation force and } \\ \text { optimal velocity. }\end{array}\end{array}$

$K_{\text {opt }}(\omega) \quad$ Same as $H_{\text {opt }}(\omega)$ after singularity for $\omega \rightarrow \infty$ is removed.

$H_{\text {red }}(\omega) \quad$ Manipulation of $H_{\text {opt }}(\omega)$ for reduced causality.

$K_{\text {red }}(\omega) \quad$ Same as $H_{\text {red }}(\omega)$ after singularity for $\omega \rightarrow \infty$ is removed.

$\omega_{1}$

Lowest frequency pole of $H_{\text {opt }}(\omega)$ or $H_{\text {red }}(\omega)$. $\tau_{1}$

$L$

RCW
Main time-constant of $H_{\text {opt }}(\omega)$ or $H_{\text {red }}(\omega)$.

Future time-horizon considered for implementation of noncausal $H_{\text {opt }}(\omega)$.

Relative capture width.

\section{INTRODUCTION}

$\mathbf{O}$ CEAN wave power can make an important contribution to the development of a more sustainable, and renewable, energy strategy in different countries worldwide [1]-[3]. In order to raise the economic performance of wave energy converters (WECs), still far from being competitive, a large scope exists for the improvement of their capacity factor through more intelligent control systems. In particular, the efficiency of WECs, of the oscillating type, can be significantly increased through an automatic control that tunes its oscillations to the incident wave elevation, in such a way to improve the power transfer from the ocean to the system.

Real-time control of WECs is based on the optimization of the system's motion for maximum wave energy absorption. The unconstrained optimal solution, namely reactive control or complex-conjugate control, specifies the conditions under which the system is always in resonance with the wave force but has some practical limitations [4], [5]. Alternative suboptimal control solutions, formulated as constrained optimization problems, have also been proposed, where the limitation imposed by the physics of the system (e.g., amplitude of motion or velocity, applicable forces) are also taken into account. In particular, some of these alternatives include latching [6], [7], where the oscillation in the system is delayed so to be in phase with the excitation from the waves, and model predictive control (MPC) [8]-[10], which handles the use of constraints.

It is acknowledged that the effectiveness of the different realtime control strategies depends on, among others, the prediction of the future wave elevation or wave excitation force acting on the system [5], [6], [8], [11]-[14]. Note that simpler suboptimal, but at the same time effective, causal control solutions, that do not require predictions, have also been proposed in [15] and [16].

The problem of short-term wave forecasting has been studied by a number of researchers. Purely stochastic time series solutions have been proposed in [17], [18], and [13], where the future wave elevation (or force/motion) is extrapolated from a combination of its past values. Particularly in [18] it is shown, on real wave measurements, how autoregressive (AR) models allow the achievement of accurate forecasts for more than 1 wave period into the future. Alternative deterministic approaches have been also investigated, where the incident wave (or force/motion) is predicted from distant measurements [12], [19]-[22]. The latter solutions can produce more accurate 
predictions for longer into the future, at the expense of more instrumentation and higher algorithm complexity. In [22], it is claimed that prediction, some 60 seconds in advance, of ship and platform motions, due to the incident wave elevation, is realized.

The prerequisites for the implementation of the aforementioned control solutions, in terms of prediction, were never formally analyzed and quantified. In addition, it is of fundamental importance to understand how long into the future predictions are required for, and if there is any particular property of the WEC that may have an influence on this time horizon. Not only is such an analysis important to judge the adequateness of the forecasting solutions, but it is also important at the design stage of a WEC, when controllability may need to be considered. Throughout the paper, the focus is put on a WEC consisting of a single floating body constrained to move in one degree of freedom. While this assumption does not limit the generality of the results, it allows for a clearer understanding of the fundamental relations between the system and the prediction requirements. The quantitative analysis is confined to unconstrained optimal control, but other control possibilities are also qualitatively discussed.

After the floating system and the control solutions are introduced in Section II, the methodology proposed for the study of the prediction requirements is presented in Section III. Results obtained for some ideal systems over a variety of sea states are then discussed in Section IV. Section V, finally, points out the main conclusive remarks.

\section{A. The System}

\section{Control System}

The WEC considered in this study consists of a generic floating body, oscillating in heave. The relative motion with respect to the sea bottom is converted into useful electricity by a power takeoff (PTO) mechanism, the detail of which is left unspecified at this stage. The motion of such a system, illustrated in Fig. 1, is described as follows:

$M \dot{v}(t)+\int_{0}^{t} z(t-\tau) v(\tau) d \tau+K_{f} v(t)+K_{s} x(t)=f_{\mathrm{ex}}(t)+f_{u}(t)$

where $M$ is the mass of the body, $v(t)$ and $x(t)$ its velocity and position, $z(t)$ is the kernel function modeling the radiation damping, $K_{f}$ a constant modeling the friction, and $K_{s}$ the buoyancy coefficient. The external forces acting on the system are the excitation force $f_{\text {ex }}(t)$ due to the incident waves, and a controllable load force $f_{u}(t)$ produced by the PTO. Note that (1) is valid under the assumption of zero initial conditions, that is $x(0)=v(0)=0$.

Linearity is assumed for all the hydrodynamic forces, so that the model of the WEC can alternatively be expressed in the frequency domain

$$
Z_{i}(\omega) V(\omega)=F_{\mathrm{ex}}(\omega)+F_{u}(\omega)
$$

where all the properties of the system are incorporated in the intrinsic mechanical impedance $Z_{i}(\omega)$ defined as

$$
Z_{i}(\omega)=B(\omega)+K_{f}+\jmath \omega\left[M+M_{a}(\omega)-\frac{K_{s}}{\omega^{2}}\right] .
$$

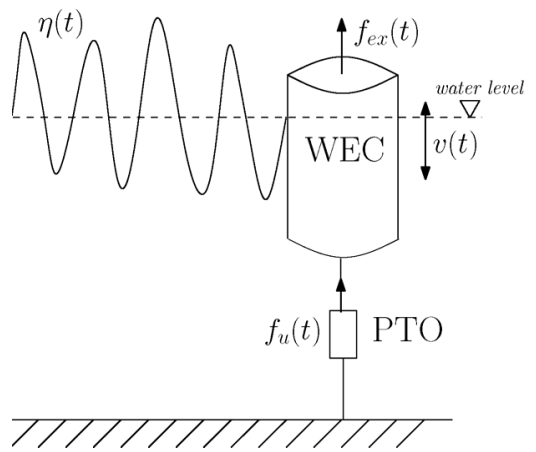

Fig. 1. Floating cylinder oscillating in heave.

In (3), the Fourier transform of the radiation kernel is expressed as $\mathcal{F}\{z(t)\}=B(\omega)+\jmath \omega M_{a}(\omega)$, in terms of radiation resistance $B(\omega)$ and added mass $M_{a}(\omega)$. Note that such a Fourier transform is only valid in a generalized sense, as $M_{a}(\omega)$ does not, in general, vanish in the limit $\omega \rightarrow+\infty$ [4].

The excitation force $f_{\text {ex }}(t)$ is the effect that the incident wave elevation $\eta(t)$ has on the system, determined by the noncausal excitation transfer function $H_{\mathrm{ex}}(\omega)$ [4]

$$
F_{\mathrm{ex}}(\omega)=H_{\mathrm{ex}}(\omega) \Xi(\omega) .
$$

Note that in (4), $\Xi(\omega)$ represents the Fourier transform of the wave elevation $\Xi(\omega)=\mathcal{F}\{\eta(t)\}$.

\section{B. Unconstrained Optimal Control}

No specifications about the PTO mechanism which actually provides the load force is made at this stage. The only assumption is that it can produce any force calculated by a control law, assumed to be of the form

$$
F_{u}(\omega)=-Z_{u}(\omega) V(\omega)
$$

where the controller $Z_{u}(\omega)$ can be thought of as the impedance realized by the PTO. Without considering any constraints on the achievable forces (we did not make any assumptions about the PTO), and without any hypothesis about the disturbance $f_{\mathrm{ex}}(t)$, the only criterion for the design of the controller is that it allows maximum energy transfer from the excitation (disturbance) to the load.

The average power $P_{u}$, absorbed at the load over the time $T$, is the time integral of the product of the load force and the system velocity

$$
P_{u}=-\frac{1}{T} \int_{0}^{T} f_{u}(t) v(t) d t .
$$

Such an expression for the average power can more conveniently be expressed in the frequency domain, by making use of Parseval's theorem [4]

$$
P_{u}=-\frac{1}{2 \pi T} \int_{0}^{+\infty}\left[F_{u}(\omega) V^{*}(\omega)+F_{u}^{*}(\omega) V(\omega)\right] d \omega .
$$

The notation $(\cdot)^{*}$ indicates the complex-conjugate operation. Note that Parseval's theorem can only be applied if the force 


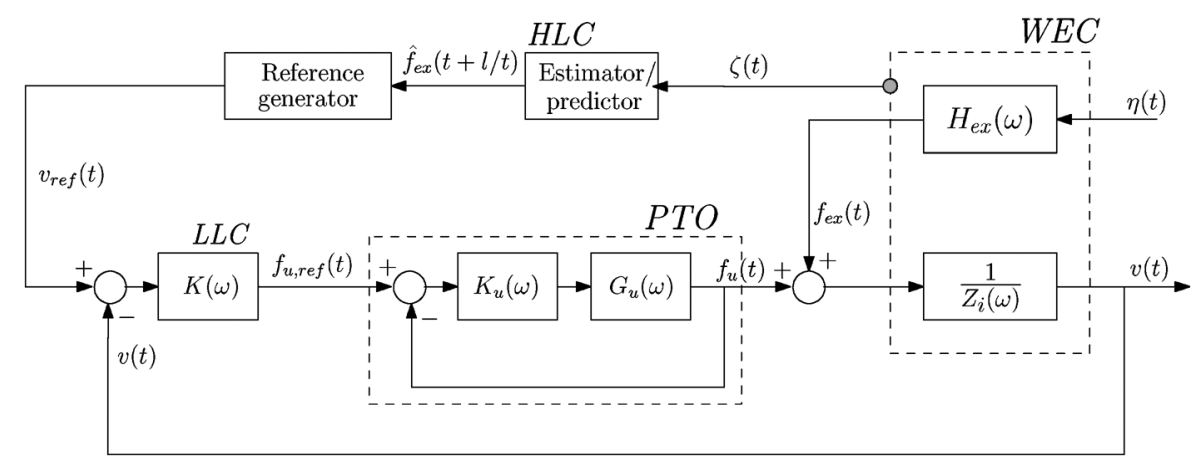

Fig. 2. General structure for a real-time controller of a WEC.

and velocity signals are supposed to be zero outside the time interval $[0, T]$. The integrand of (7) is proportional to the average power absorbed at each frequency

$$
P_{u}(\omega)=F_{u}(\omega) V^{*}(\omega)+F_{u}^{*}(\omega) V(\omega) .
$$

Using the dynamic equation in (2), and considering the control law (5), the velocity and the load force can be written as functions of the external disturbance only

$$
\begin{aligned}
V(\omega) & =\frac{1}{Z_{i}(\omega)+Z_{u}(\omega)} F_{\mathrm{ex}}(\omega) \\
F_{u}(\omega) & =-\frac{Z_{u}(\omega)}{Z_{i}(\omega)+Z_{u}(\omega)} F_{\mathrm{ex}}(\omega)
\end{aligned}
$$

so that the average absorbed power, from (8), becomes

$$
P_{u}(\omega)=-\frac{Z_{u}(\omega)+Z_{u}^{*}(\omega)}{\left[Z_{i}(\omega)+Z_{u}(\omega)\right]\left[Z_{i}(\omega)+Z_{u}(\omega)\right]^{*}}\left|F_{\mathrm{ex}}(\omega)\right|^{2} .
$$

Note that the minus sign in (6)-(11) is due to the fact that the excitation power entering the system is considered to be positive, so that the power absorbed at the load (exiting the system) will, therefore, be negative.

Maximization of (11), with respect to $Z_{u}(\omega)$, yields the optimal control law producing the load force and system's velocity that allow maximum power transfer from the waves to the load [4]

$$
P_{u}(\omega)=\max \quad \Leftrightarrow \quad Z_{u}(\omega)=Z_{u, \mathrm{opt}}(\omega)=Z_{i}^{*}(\omega)
$$

and this is independent of the power spectral distribution of the disturbance $\left|F_{\mathrm{ex}}(\omega)\right|^{2}$. The optimal control law in (12) is termed, in the wave energy literature, complex-conjugate control, as the load impedance required in order to produce the optimal force is the complex-conjugate of the intrinsic impedance of the system.

The name reactive control is also used, to highlight the presence of a reactive power and the necessity to inject energy into the system during part of the cycle. This requirement of a reversible power flow has major implications on the practical implementation of reactive control. Although maximum mechanical energy is ensured, in fact, losses in the bidirectional flow implemented by a nonideal PTO mechanism may in fact result in a poor overall efficiency of the system, if one considers the actual useful (e.g., electrical) energy produced.
The optimal controller in (12) is not realizable in practice because it is noncausal [4]. Predictions of the excitation force can be utilized, however, to estimate the optimal velocity from the following noncausal relation:

$$
V_{\mathrm{opt}}(\omega)=\frac{1}{2 B(\omega)+2 K_{f}} F_{\mathrm{ex}}(\omega)
$$

which is easily derived from (9) and (12). The system can then be forced to follow this reference velocity through a feedback control loop. The structure of the proposed real-time controller is shown in Fig. 2, where the noncausal condition (13) is implemented in a higher level control (HLC) that decides the optimal reference velocity to be imposed on the WEC. Estimation of the excitation force, which is not directly measurable, is required [8], along with its prediction.

\section{Other Control Approaches}

Other control architectures have been proposed, where the load force is analytically determined from the excitation force or from the velocity, using (10) and (12). The velocity of the floating system is then controlled, in a feed-forward fashion, by applying the calculated PTO force [14], [23], [24]. Such solutions, in the authors' opinion, may lose their effectiveness in a real-world implementation, where inaccuracies in the model (nonlinearities, uncertainties in the parameters, or prediction errors) could lead to poor control actions.

As mentioned in the introduction, practical constraints usually limit the applicability of reactive control. In particular, the condition in (12) may require infeasible amplitudes or velocities of the motion, as well as forces significantly bigger than the PTO mechanism can provide, or that the system can bear. Another constraint could be the inability to provide reactive power from the load, if the PTO is purely passive. This is why alternative control strategies have been investigated, where the control problem is treated as a real-time constrained optimization problem and an optimal reference trajectory for the system's variables (velocity and PTO force) is generated from (future) knowledge of the excitation force. Most common control strategies of this type are latching (passive) [6], [25] and model predictive control (MPC) [8]-[10].

While the optimal velocity given by reactive control, as in (13), is based on the minimization of the average power over an infinite time horizon, MPC, on the other hand, optimizes the operation of the system (velocity and/or force) over a finite future 
horizon. In particular, at each time step $k$, the absorbed power, over a future time horizon $L$, is maximized

$$
J(k)=\sum_{j=1}^{L} f_{u}(k+j) v(k+j)=\sum_{j=1}^{L} P_{u}(k)
$$

possibly subject to constraints on the system's variables.

Due to a number of reasons, however, the scope of the present study will be limited to reactive control, in Sections III and IV. In general, the solution of constrained optimization problems would converge towards reactive control when the system's operating conditions are well within the constraints. In addition, when the limits of the constrained region are approached, it is not usually possible to derive an analytical solution and the prediction requirements can only be determined numerically and on a case by case basis. On the other hand, restraining the analysis to reactive control allows an understanding of the basic links that exist between the fundamental properties of a floating system (radiation and excitation) and its prediction requirements.

\section{Measures of Noncausality AND PRedictability}

This section outlines a methodology for the quantification of the prediction requirements concerning reactive control, structured as in Fig. 2. After a fundamental discussion of the noncausality of the control in Section III-A, quantifications of the prediction requirements and of the predictability are proposed in Sections III-B and III-C, respectively. The possibility of manipulating the control to achieve a reduction in the prediction requirements is also discussed in Section III-D.

\section{A. Analysis of Noncausality}

The noncausality of the control strategy described in Section II, and shown in Fig. 2, is encapsulated by the following relationship:

$$
V_{\mathrm{opt}}(\omega)=\frac{1}{2 B(\omega)+2 K_{f}} F_{\mathrm{ex}}(\omega) \triangleq H_{\mathrm{opt}}(\omega) F_{\mathrm{ex}}(\omega)
$$

which can be equivalently written in the time domain as

$$
v_{\mathrm{opt}}(t)=\int_{-\infty}^{t} h_{\mathrm{opt}}(\tau) f_{\mathrm{ex}}(t-\tau) d \tau
$$

where $h_{\mathrm{opt}}(t)$ is the inverse Fourier of $H_{\mathrm{opt}}(\omega)$

$$
h_{\text {opt }}(t)=\mathcal{F}^{-1}\left\{\frac{1}{2 B(\omega)+2 K_{f}}\right\}=\mathcal{F}^{-1}\left\{H_{\text {opt }}(\omega)\right\} \text {. }
$$

When $\omega \rightarrow \infty$, the radiation resistance $B(\omega)$ goes to zero and, therefore, $H_{\text {opt }}(\omega)$ tends to the constant value $1 / 2 K_{f}$. This means that the transform relationship in (17) is only valid in a generalized sense and, more correctly

$$
\begin{aligned}
h_{\mathrm{opt}}(t) & =\frac{1}{2 K_{f}} \delta(t)+\mathcal{F}^{-1}\left\{H_{\mathrm{opt}}(\omega)-\frac{1}{2 K_{f}}\right\} \\
& =\frac{1}{2 K_{f}} \delta(t)+k_{\mathrm{opt}}(t)
\end{aligned}
$$

where $\delta(t)$ is the Dirac delta function. The inverse Fourier transform in the right-hand term of (18), yielding the function

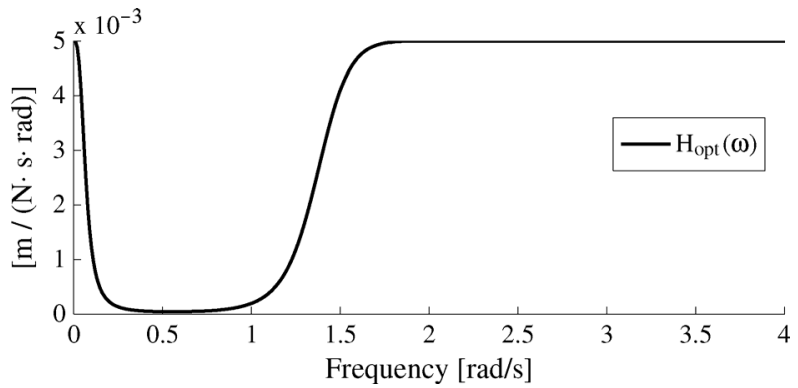

Fig. 3. Noncausal reference-generator transfer function between excitation force and optimal velocity. It is a real and even function of the frequency.

$k_{\text {opt }}(t)$, is now well defined. Note, as far as noncausality is concerned, $h_{\text {opt }}(t)$ or $k_{\text {opt }}(t)$ have the same characteristics, as they only differ at time $t=0$.

The impulse response $h_{\mathrm{opt}}(t)$ is real and even (the Fourier transform is real and even) and tends to zero for infinite time (the Fourier transform tends to a constant value for $\omega=0$ ). The decay to zero is dominated, in general, by an exponential with a time constant $\tau_{1}$ which is the reciprocal of the lowest frequency pole of the corresponding transfer function.

Due to the presence of the radiation resistance $B(\omega), H_{\mathrm{opt}}(\omega)$ is only known numerically for some frequencies, and an explicit finite-order representation is not immediately available. From the behavior shown in Fig. 3, valid for systems with a singlepeaked radiation, it is, however, straightforward to identify the lowest frequency pole. The function $H_{\mathrm{opt}}(\omega)$ can, in fact, be seen as a low-pass filter cascaded with a high-pass filter, where the attenuation band corresponds to the resonance band of the system's radiation function. The lowest frequency pole, namely $\omega_{1}$, can be estimated as the cutoff frequency (at $-3 \mathrm{~dB}$ ) of the low-pass filter.

The time constant $\tau_{1}=1 / \omega_{1}$ is the dominant parameter that influences the decay to zero of $h_{\mathrm{opt}}(t)$, and consequently the noncausality of the control action. As an example, Fig. 4 shows the relation between $\tau_{1}$ and $\omega_{1}$ for two different floating systems.

\section{B. Prediction Requirements}

In Section III-A, a parameter strictly connected to the radiation of the floating system $\tau_{1}$ was identified as a measure of the noncausality of its control system. It would be interesting to see if a direct proportionality exists between $\tau_{1}$ and the actual forecasting horizon required for an effective approximation of the convolution in (16), for the generation of the optimal velocity reference.

The required prediction horizon is determined from a range of suboptimal oscillation velocities, calculated from the integral in (16), truncated at different times $\tau=-L$

$$
v_{\mathrm{opt}}^{L}(t)=\int_{-L}^{L} h_{\mathrm{opt}}(\tau) f_{\mathrm{ex}}(t-\tau) d \tau
$$

so that future values for the excitation force only up to $f_{\mathrm{ex}}(t+$ $L$ ) (supposed to be perfectly known) are included. Note that the causal part of the integral is also truncated to maintain the 

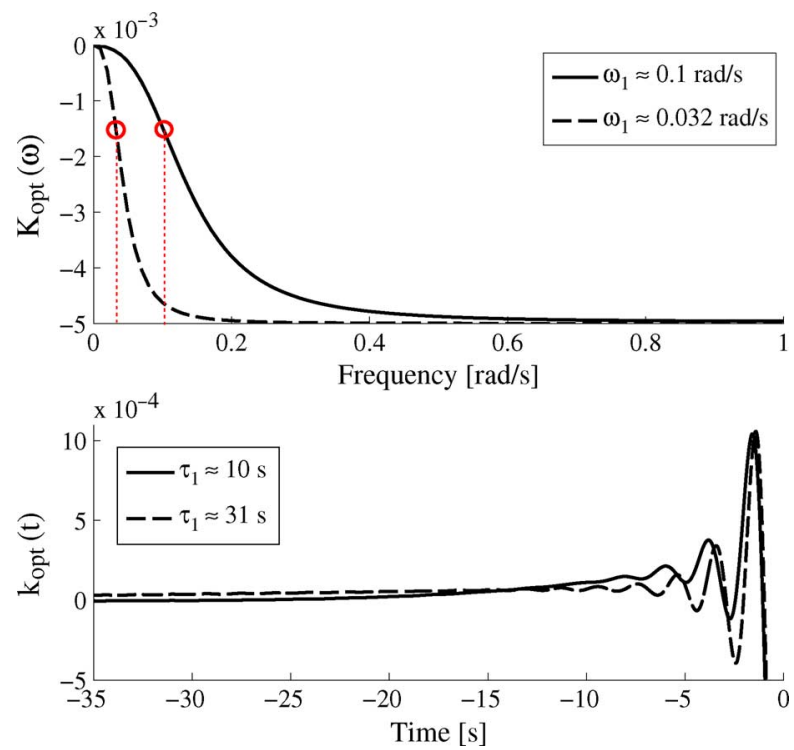

Fig. 4. Relation between lowest frequency pole and noncausality of the impulse response. $K_{\mathrm{opt}}(\omega)$ and $k_{\mathrm{opt}}(t)$ calculated for two heaving buoys.

evenness of the impulse response. In the extreme case of $L=0$, the integral in (19) becomes causal and no future values of the excitation force are utilized.

The velocity $v_{\mathrm{opt}}^{L}(t)$ is then ideally imposed onto the system, using a load force that can be directly derived from (1). The relative capture width ( $\mathrm{RCW}$ ), obtained with the velocity thus calculated (and with the required PTO force), is

$$
\operatorname{RCW}(L)=\frac{P_{u}(L)}{P_{w} \cdot D}
$$

where $P_{u}(L)$ is the absorbed power, calculated from (6), $D$ is the width of the device (the diameter in the case of a cylinder or sphere), and $P_{w}$ is the wave power per unit width [4]

$$
P_{w}=\frac{1}{2} \rho g^{2} \int_{0}^{+\infty} \frac{\Xi(\omega) \Xi^{*}(\omega)}{\omega} d \omega .
$$

In (21), $g$ is the acceleration due to gravity and $\rho$ is the water density. Also, deep water is assumed, that is $k h \gg 1$ [4], where $k$ is the wave number and $h$ is the water depth.

Intuitively, it is expected that $\mathrm{RCW}(L)$ increases when larger values of $L$ are considered, but only up to a critical future horizon, beyond which the power gain from considering extra future information of the excitation force is negligible. Such a critical future horizon will be proportional to the time constant $\tau_{1}$.

\section{Prediction of the Excitation Force}

The ability to predict the excitation force is tested with linear autoregressive (AR) models, whose identification and estimation is operated accordingly to the methodology presented in [18]. In particular, at instant $k$, the $l$-step ahead prediction, $\hat{f}_{\mathrm{ex}}(k+l \mid k)$, is calculated as

$$
\hat{f}_{\mathrm{ex}}(k+l \mid k)=\sum_{i=1}^{n} a_{i}(k) \hat{f}_{\mathrm{ex}}(k+l-i \mid k)
$$

where $a_{i}, i=1, \ldots, n$ are the coefficients of the AR model, of order $n$ and, obviously, $\hat{f}_{\mathrm{ex}}(k+l-i \mid k)=f_{\mathrm{ex}}(k+l-i)$ if $k+l-i \leq k$ (information acquired, no need for prediction).

The performance of the prediction algorithm is measured in terms of the following index of goodness-of-fit:

$$
\mathcal{F}(l)=\left(1-\frac{\sqrt{\sum_{k}\left[f_{\mathrm{ex}}(k+l)-\hat{f}_{\mathrm{ex}}(k+l \mid k)\right]^{2}}}{\sqrt{\sum_{k} f_{\mathrm{ex}}(k)^{2}}}\right) \cdot 100 .
$$

In [18] it was shown how AR models allow for accurate predictions of the wave elevation, at low frequencies, for more than one wave period into the future. From (4), the excitation force represents the wave elevation low-pass filtered by the function $H_{\text {ex }}(\omega)$, which encloses the excitation properties of the floating system.

The stochastic nature of the AR model implies that the achievable prediction horizon cannot exceed the intrinsic correlation of the signal, as the physics of the phenomenon (wave propagation laws) is not exploited. As a consequence, and based on the fact that the correlation in a signal is in general inversely proportional to its bandwidth, it may be expected that the ability to predict the excitation force, by using a stochastic model is related to properties of the sea state [18] (peak frequency and bandwidth), but also to the system's filtering properties [26], specified by the function $H_{\mathrm{ex}}(\omega)$.

For a given sea state, systems with a narrower excitation bandwidth will experience lower frequency waves and, therefore, will allow for longer prediction horizons, in seconds.

\section{Reduction of Prediction Requirements}

In some situations, it may happen that the prediction requirements, determined from Section III-B, are beyond the ability of the prediction model. One solution could be the adoption of complex forecasting solutions, making use of expensive instrumentation and of more computationally intensive models [22]. A critical evaluation of the noncausality of $H_{\text {opt }}(\omega)$, which also takes into account the typical frequency distribution of the excitation force, offers a much simpler alternative.

For a number of reasons, the wave excitation force is always contained within the stopband of $H_{\mathrm{opt}}(\omega)$ :

1) Waves do not appear at frequencies below $0.2-0.3 \mathrm{rad} / \mathrm{s}$ (30-40 s).

2) Waves at higher frequencies than the attenuation band of $H_{\text {opt }}(\omega)$ are filtered out by the excitation transfer function, $H_{\mathrm{ex}}(\omega)$. There exists, in fact, a correspondence between the radiation and excitation bandwidth (based on the Haskind relation) [4], [26].

3) If the system is well-suited to the location of deployment, its radiation response shall roughly match most of the sea states, and this corresponds to the attenuation band of $H_{\mathrm{opt}}(\omega)$, as discussed in Section III-B.

Based on these considerations, it may be argued that the reference-generator will mostly work at frequencies within the attenuation band of $H_{\mathrm{opt}}(\omega)(0.4-1 \mathrm{rad} / \mathrm{s}$ in Fig. 3). As a consequence, there exists the possibility of manipulating the shape of $H_{\text {opt }}(\omega)$ in order to reduce its noncausality, while at the same time maintaining its effectiveness in most sea conditions. 


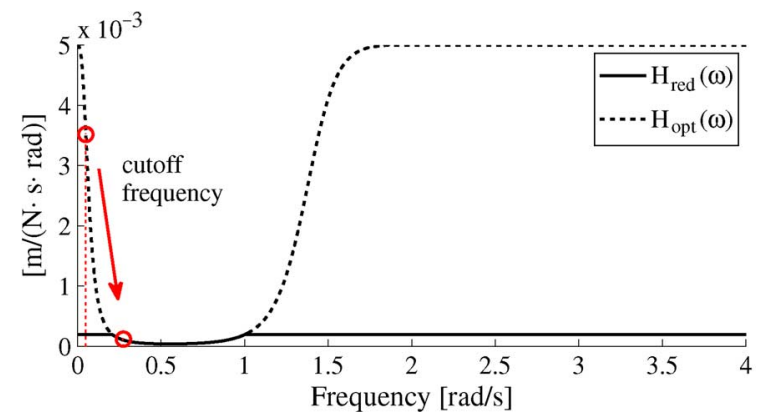

(a)

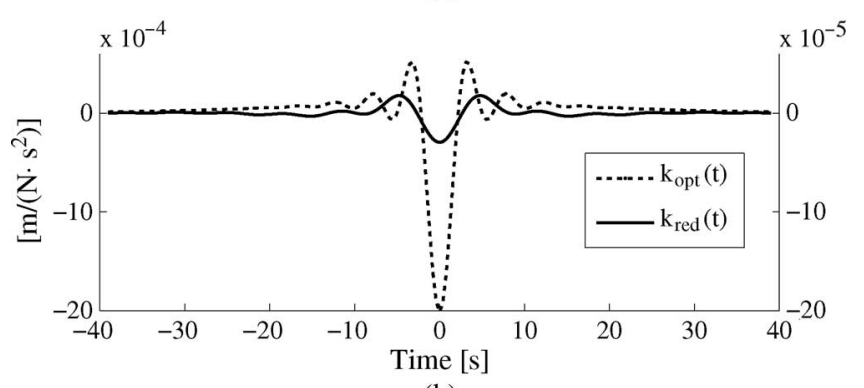

(b)

Fig. 5. Manipulation of $H_{\mathrm{opt}}(\omega)$, resulting in $H_{\text {red }}(\omega)$, such that the lowest frequency pole is moved rightward, thus decreasing the dominant time constant of the correspondent impulse response. Note that the impulse response $k_{\mathrm{opt}}(t)$ instead of $h_{\mathrm{opt}}(t)$ is shown, as from (18).

In Section III-B, the noncausality was related to the time constant $\tau_{1}$, which is the reciprocal of the pole at the lowest frequency of $H_{\mathrm{opt}}(\omega)$. A possible intuitive manipulation may, therefore, consist of lowering the gain of $H_{\mathrm{opt}}(\omega)$ outside the attenuation band, where its input (the excitation force) will not usually appear. This would move the first pole towards higher frequencies, thus reducing the time constant $\tau_{1}$. As an example, consider Fig. 5(a), where the original $H_{\text {opt }}(\omega)$ is modified to a new function termed $H_{\text {red }}(\omega)$. As a result, the impulse response $h_{\text {red }}(t)$, which is still real and even, decays much more quickly to zero than $h_{\mathrm{opt}}(t)$, as shown in Fig. 5(b).

Note that the modification proposed here is not rigorous, in any sense, and is only based on qualitative considerations. It is not within the scope of the paper to find the best reshaping of $H_{\text {opt }}(\omega)$ which minimizes the noncausality and at the same time maximizes the approximation of the optimality condition.

A very important remark should also be mentioned. From Figs. 3 and 5(a), it may be noted that, over its attenuation band, the function $H_{\text {opt }}(\omega)$ is quite flat. This observation may suggest that a constant approximation of $H_{\text {opt }}(\omega)$ could be close to optimal in most sea states. The possibility of approximating $H_{\text {opt }}(\omega)$ with a constant value is investigated in more detail in [16].

\section{Results}

The wave data utilized for the analysis of the prediction requirements was provided by the Irish Marine Institute and comes from real measurements collected from a data buoy deployed off the West coast of Ireland, in the Belmullet wave energy test site, at approximately $54^{\circ} 13^{\prime} \mathrm{N}, 10^{\circ} 8^{\prime} \mathrm{W}$. The data consists of a number of selected 30-min sets, sampled at $1.28 \mathrm{~Hz}$, as in Fig. 6(b), covering a variety of sea condition in the year 2010. Fig. 6(a) shows the distribution of the peak

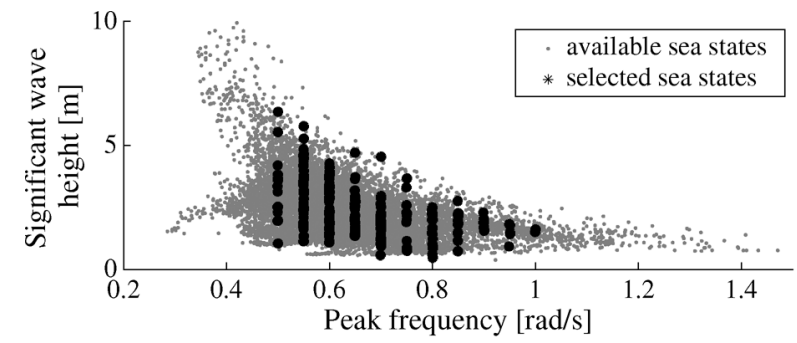

(a)

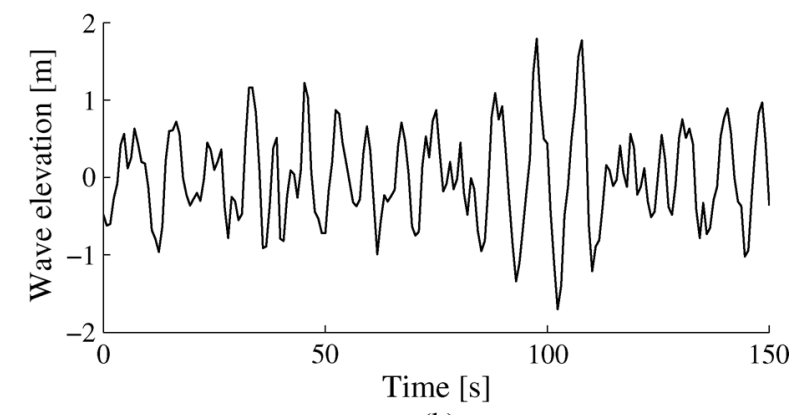

(b)

Fig. 6. Selected wave data: (a) overall statistics of selected data sets; (b) sample data set. Location is Belmullet, off the West coast of Ireland.

frequency, $\omega_{p}$, and significant wave height $H_{s}$ of the selected data sets.

The wave energy conversion system consists of a bottomreferenced floating body constrained to move in the heave direction only, as modeled in Section II-A. Both cylinders and spheres are considered, with a range of values for the radius and the height. The detail of the geometries is specified in Table I, where $R$ : radius; $h$ : draught at rest; $M$ : submerged mass at rest; $\omega_{0}$ : resonance frequency; $\Delta \omega$ : bandwidth of the radiation resistance $B(\omega) ; \tau_{1}$ : main time constant of the control $h_{\mathrm{opt}}(t)$. As the radius of the sphere increases, the bandwidth and the resonance frequency get smaller, while the dominant time constant of the noncausal control $\tau_{1}$ increases. Regarding the cylinders, the time constant $\tau_{1}$ is mainly affected by the radius, while it only changes slightly for different heights.

Based on the methodology proposed in Section III-B, reactive control is implemented with the (truncated) noncausal law in (19), where only future knowledge of the excitation force up to $L$ seconds into the future is included. The resulting performance $\mathrm{RCW}(L)$ is calculated for a range of values of the lead time $L$. Fig. 7(a) shows the behavior of $\operatorname{RCW}(L)$ for the three spheres of Table I, in a particular sea state. As expected, the performance improves if more future information about the excitation force is included in the control. At some point, however, the optimum is approached and no further benefit is gained from extra knowledge of the future. This critical future horizon depends on the geometry and increases with $\tau_{1}$, as expected. Note that the optimal value of RCW changes with the size of the device, in accordance with the general theory of axisymmetric heaving buoys [27].

In Fig. 7(b), the average lead time $L$, for which $\mathrm{RCW}(L)>$ 0 , over all the considered sea states (about 200), is plotted against the time constant $\tau_{1}$ corresponding to the case of the three spheres. Interestingly, the relation between the future horizon required and $\tau_{1}$ is quite linear (the variance of the residuals of a linear fit $\sim 0.78$ ). Besides, the standard deviation 


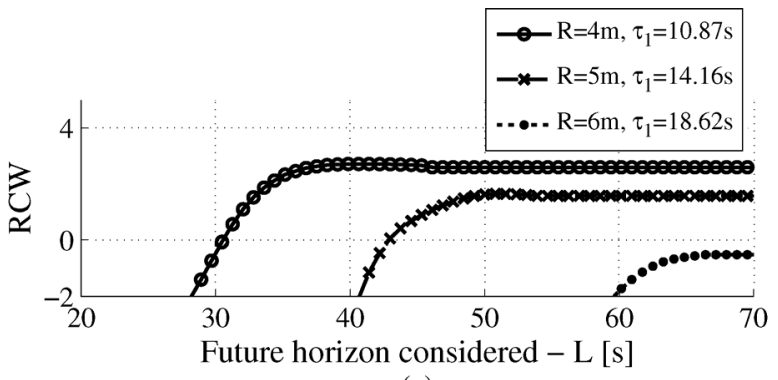

(a)

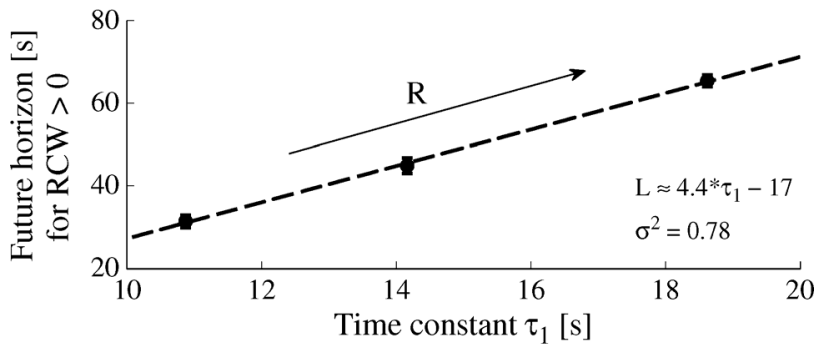

(b)

Fig. 7. Performance of reactive control, applied to heaving spheres, when only limited future information of the excitation force, up to $L$ seconds in the future, is considered. (a) Detail for a specific sea state. (b) Required horizon averaged over all sea states.

TABLE I

Geometry of Heaving BuOYs. $C$ : Cylinder; $S$ : SpHere

\begin{tabular}{l||ccc||c|c|c|}
\hline & $\begin{array}{c}R \\
{[\mathrm{~m}]}\end{array}$ & $\begin{array}{c}h \\
{[\mathrm{~m}]}\end{array}$ & $\begin{array}{c}M \\
{\left[\mathrm{~kg} \times 10^{5}\right]}\end{array}$ & $\begin{array}{c}\omega_{0} \\
{[\mathrm{rad} / \mathrm{s}]}\end{array}$ & $\begin{array}{c}\Delta \omega \\
{[\mathrm{rad} / \mathrm{s}]}\end{array}$ & $\begin{array}{c}\tau_{1} \\
{[\mathrm{~s}]}\end{array}$ \\
\hline$S_{1}$ & 4 & 4 & 1.38 & 1.63 & 1.60 & 10.87 \\
$S_{2}$ & 5 & 5 & 2.70 & 1.43 & 1.45 & 14.16 \\
$S_{3}$ & 6 & 6 & 4.66 & 1.32 & 1.37 & 18.62 \\
\hline$C_{1}$ & 3 & 4 & 0.93 & 1.40 & 1.03 & 10.00 \\
$C_{2}$ & 3 & 8 & 1.86 & 1.08 & 0.79 & 9.97 \\
$C_{3}$ & 3 & 12 & 2.80 & 0.92 & 0.66 & 9.94 \\
$C_{4}$ & 3 & 16 & 3.73 & 0.82 & 0.59 & 9.91 \\
$C_{5}$ & 3 & 20 & 4.66 & 0.74 & 0.53 & 9.89 \\
$C_{6}$ & 5 & 4 & 2.59 & 1.23 & 0.98 & 19.80 \\
$C_{7}$ & 5 & 8 & 5.18 & 1.02 & 0.75 & 19.80 \\
$C_{8}$ & 5 & 12 & 7.77 & 0.89 & 0.63 & 19.76 \\
$C_{9}$ & 5 & 16 & 10.40 & 0.79 & 0.56 & 19.7 \\
$C_{10}$ & 5 & 20 & 12.90 & 0.72 & 0.51 & 19.76 \\
$C_{11}$ & 7 & 4 & 5.07 & 1.14 & 0.95 & 31.06 \\
$C_{12}$ & 7 & 8 & 10.10 & 0.98 & 7.2 & 30.96 \\
$C_{13}$ & 7 & 12 & 15.20 & 0.85 & 0.62 & 30.96 \\
$C_{14}$ & 7 & 16 & 25.40 & 0.70 & 0.54 & 30.96 \\
$C_{15}$ & 7 & 20 & 31.71 & 0.64 & 0.50 & 30.9 \\
\hline
\end{tabular}

related to each mean value (dot) in Fig. 7(b) is very small (bar centered around each dot), which confirms the independence of the prediction requirements from the specific wave conditions.

In the case of the cylinders, there are two parameters to be considered: the height and the radius. The time constant $\tau_{1}$ is mainly influenced by the radius, so that the prediction requirements are not expected to be significantly affected by variations in height. Fig. 8 shows the required prediction horizon $L$, such that $\mathrm{RCW}(L)>0$, averaged over all the considered sea states, against the time constant $\tau_{1}$, for each cylinder. As for the spheres, the forecasting horizon required increases with $\tau_{1}$ (and with the radius). In addition, for a given height, the relation is linear (variance of the residuals of a linear fit $\sim 0.04$ ). To a lesser extent, changes in height also affect the prediction requirements and the trend is consistent: the cylinders are less demanding in terms of prediction when their height increases. The parameter

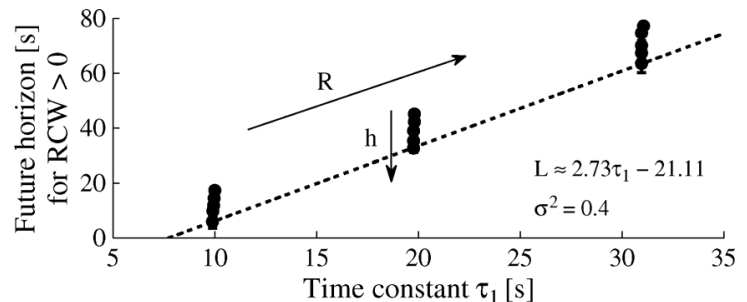

Fig. 8. Future prediction horizon $L$ required for effective implementation of reactive control, applied to heaving cylinders.

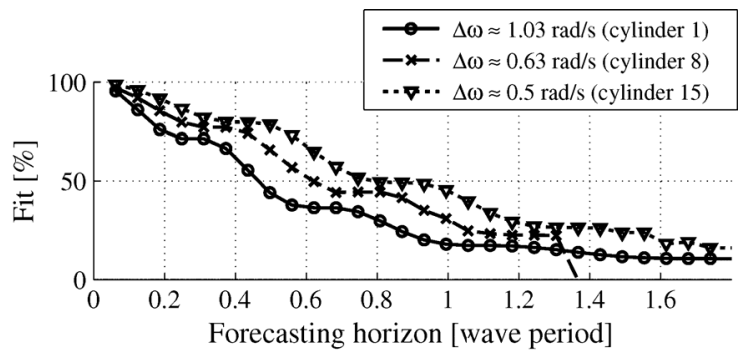

(a)

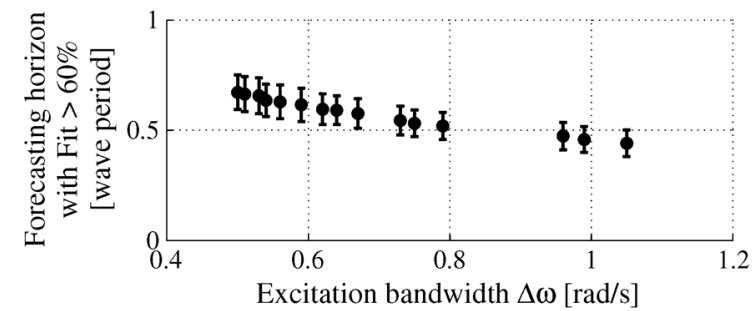

(b)

Fig. 9. Accuracy of AR prediction models applied to the excitation force produced on heaving cylinders.

$\tau_{1}$ remains, though, the most critical in relation to the prediction requirements.

Having quantified the required forecasting horizon, the ability to predict the excitation force is discussed. As proposed in Section III-C, linear AR forecasting models are utilized, identified and estimated as in [18]. Focus is put on the cylinders of Table I, and predictions are calculated for all the selected wave data. The accuracy of the prediction, at each lead time $L$, is measured with the index $\mathcal{F}(L)$, from (23). In Fig. 9(a), the behavior of $\mathcal{F}(L)$ for the excitation force produced by a particular sea state, on three cylinders (with different bandwidths), is shown. The forecasting horizon is expressed as a fraction of the peak period. The effectiveness of the prediction obviously decreases with the forecasting horizon, and the level of accuracy is higher for systems with narrower bandwidth, as expected [26]. In Fig. 9(b), the forecasting horizon $L$, for which $\mathcal{F}(L)>60 \%$, averaged over all the sea states, is shown against the bandwidth of $H_{\mathrm{ex}}(\omega)$. The predictability clearly tends to decrease with increasing bandwidth. Note also that the standard deviation is relatively large, due to the fact that the properties of the waves also have a significant effect on the predictions [18].

In general, however, less than 1 wave period in the future (about 6 to $15 \mathrm{~s}$ at the considered location) can be predicted with a $\mathcal{F}>60 \%$. In comparison with the requirements, well above $10 \mathrm{~s}$ in most cases (Figs. 7 and 8), the performance of the predictor is quite poor.

The possibility of manipulating the control, such that the noncausality is reduced, was proposed in Section III-D. In- 

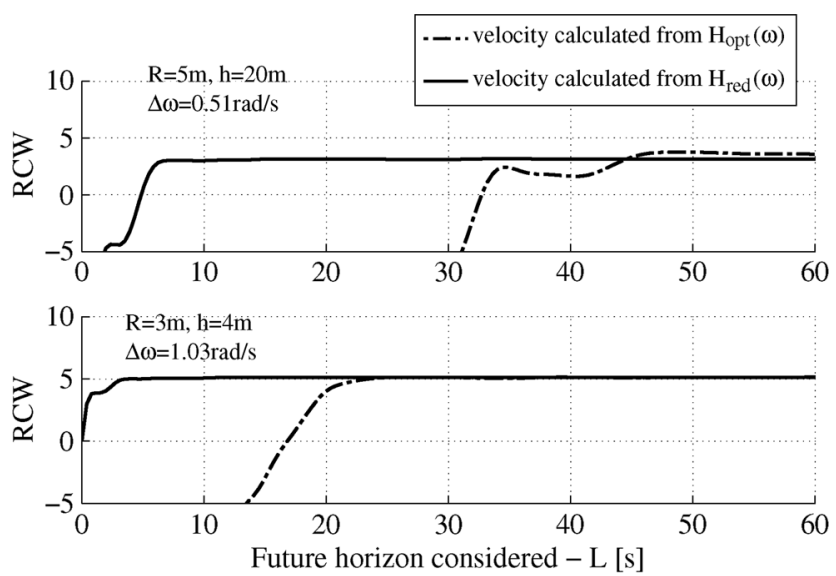

(a)

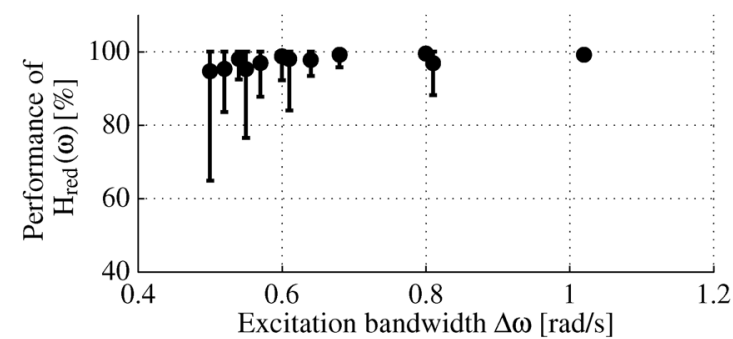

(b)

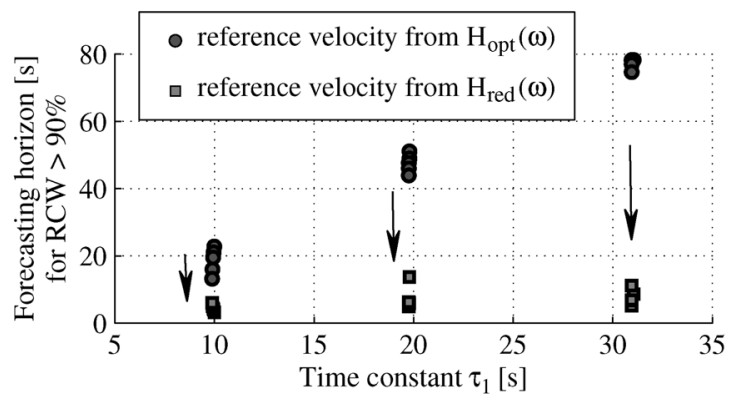

(c)

Fig. 10. Reduction of prediction requirements through manipulation of the noncausal control law: (a) Detail on a specific sea state. (b) Max RCW, when using $H_{\text {red }}(\omega)$, as percentage of ideal RCW, obtained with $H_{\text {opt }}(\omega)$. (c) Average over all sea states.

tuitive modifications to the reference generator $H_{\mathrm{opt}}(\omega)$ are applied, as shown in the example of Fig. 5(a). As a result of implementing reactive control based on the modified $H_{\text {red }}(\omega)$, Fig. 10(a) shows the behavior of $\operatorname{RCW}(L)$ for two different systems, cylinders $C_{1}$ and $C_{10}$ of Table I. A stark reduction of the prediction horizon required for an effective implementation of reactive control is achieved. In addition, the performance drop is minor in most cases. The deviation from the optimal performance, in particular, will be affected by the bandwidth of the system (flatness of $H_{\mathrm{opt}}(\omega)$ ) and by the excitation force spectral distribution, as noted in Section III-D. Fig. 10(b) shows the distribution, with the bandwidth of the system, of the maximum RCW achievable, when $H_{\text {red }}(\omega)$ is adopted, as percentage of the RCW obtained from ideal reactive control. The average (dot) performance is usually well above $80 \%$, and increases with the bandwidth of the system. The standard deviation (bar centered around dot) is quite large only for very narrow-banded systems. Note that $H_{\text {red }}(\omega)$ was only qualitatively derived from $H_{\mathrm{opt}}(\omega)$ and lower performance losses could be achieved through an optimization that takes into account the spectral distribution of the sea states, as also mentioned in Section III-D.

By applying the procedure to all the cylinders, and calculating the prediction requirements in all the sea states, the results of Fig. 10(c) are obtained. The prediction requirements are significantly reduced to an horizon shorter than $10 \mathrm{~s}$, which is well within the ability of stochastic wave forecasting models, as shown in Fig. 9 and in [18]. Also, it is interesting to note that the differences between the cylinders are quite flattened, so that no geometry seems to be particularly advantageous in terms of required forecasting horizon.

\section{CONCLUSION}

A study of the prediction requirements in real-time control of WECs was presented. Focus was put on unconstrained reactive control, which allowed the characterization of the relation between such requirements and some fundamental properties of the floating system.

A time constant, denoted $\tau_{1}$, related to the shape of the radiation resistance of a floating system, was identified as the most critical parameter influencing the noncausality of the control law. From time-domain simulations of different heaving buoys (spheres and cylinders), over a wide variety of real wave conditions, it was verified that the relation between $\tau_{1}$ and the future horizon required for an effective implementation of reactive control is practically linear.

The predictability of the excitation force was also determined using stochastic AR models that were already successfully adopted for the prediction of the wave elevation [18]. Floating systems with narrower bandwidth were found to allow better prediction of the excitation force, as already found in [26]. However, accurate predictions were obtained for lead times between 0.5 and 1 wave period, which is well below the requirements for most systems (well above $20 \mathrm{~s}$ ).

Simple manipulation of the control, based on prior knowledge of the excitation force spectral distribution, was also proposed for the reduction of the prediction requirements. Such reshaping of the noncausal control law allows a significant decrease in the prediction requirements, so that they fall within the range of predictability achievable with simple stochastic predictors. Minor performance losses, in terms of energy capture, are verified in most sea states. The possibility of making the control law causal was also proposed, and this is investigated in more detail in [16].

In order to maintain the focus of the paper on the prediction requirements, effects of the prediction errors on the control performance were not investigated. Future work will, therefore, involve the quantification of the mapping of the excitation force prediction error on the reference velocity, and on the design of a lower level control loop that could possibly reject unwanted (erroneous) components in the reference velocity. Preliminary work on such a topic can be found in [28].

\section{ACKNOWLEDGMENT}

The authors would like to thank the Irish Marine Institute for providing the wave data from the Belmullet test site. 


\section{REFERENCES}

[1] D. Halamay, T. Brekken, A. Simmons, and S. McArthur, "Reserve requirement impacts of large-scale integration of wind, solar and ocean wave power generation," IEEE Trans. Sustain. Energy, vol. 2, no. 3, pp. 321-328, Jul. 2011.

[2] F. Fusco and J. Ringwood, "Variability reduction through combination of wind and waves: A irish case study," Energy, vol. 35, pp. 314-325, 2010.

[3] X. Yang, Y. Song, G. Wang, and M. Wang, "A comprehensive review on the development of sustainable energy strategy and implementation in China," IEEE Trans. Sustain. Energy, vol. 1, no. 2, pp. 57-65, Jul. 2010.

[4] J. Falnes, Ocean Waves and Oscillating Systems. Cambridge, U.K.: Cambridge Univ. Press, 2002.

[5] J. Falnes, "A review of wave-energy extraction," Marine Structures, vol. 20, pp. 285-201, 2007

[6] A. Babarit and A. Clement, "Optimal latching control of a wave energy device in regular and irregular waves," Appl. Ocean Res., vol. 28, pp. 77-91, 2006.

[7] K. Fuat, "Time domain prediction of power absorption from ocean waves with latching control," Renew. Energy, vol. 35, no. 2, pp. 423-434, 2010.

[8] G. Bacelli, J.-C. Gilloteaux, and J. Ringwood, "A predictive controller for a heaving buoy producing potable water," in Proc. Eur. Control Conf. (ECC 2009), Budapest, Hungary, 2009, pp. 3755-3760.

[9] J. Cretel, A. W. Lewis, G. Lightbody, and G. P. Thomas, "An application of model predictive control to a wave energy point absorber," in Proc. IFAC Conf. Control Methodologies and Technology for Energy Efficiency (CMTEE 2010), Portugal, 2010.

[10] J. Hals, J. Falnes, and T. Moan, "Constrained optimal control of a heaving buoy wave-energy converter," J. Offshore Mech. Arctic Eng., vol. 133, pp. 011401-1-011401-15, 2011.

[11] A. Price, D. Forehand, and A. Wallace, "Time-span of future information necessary for theoretical acausal optimal control of wave energy converters," in Proc. Eur. Control Conf. (ECC), Budapest, Hungary, 2009, pp. 3761-3766

[12] M. R. Belmont, "Increases in the average power output of wave energy converters using quiescent period predictive control," Renew. Energy, vol. 35 , no. 12 , pp. $2812-2820,2010$.

[13] M. P. Shoen, J. Hals, and T. Moan, "Wave prediction and robust control of heaving wave energy devices for irregular waves," IEEE Trans. Energy Convers., vol. 26, no. 2, pp. 627-638, Jun. 2011

[14] J. Hals, J. Falnes, and T. Moan, "A comparison of selected strategies for adaptive control of wave energy converters," J. Offshore Mech. Arctic Eng., vol. 133, no. 3, pp. 031101-1-031101-12, 2011.

[15] M. Lopes, J. Hals, R. Gomes, T. Moan, L. Gato, and A. Falcao, "Experimental and numerical investigation of non-predictive phase-control strategies for a point-absorbing wave energy converter," Ocean Eng., vol. 36, no. 5, pp. 386-402, 2009.

[16] F. Fusco and J. Ringwood, "Suboptimal causal reactive control of wave energy converters using a second order system model," in Proc. Int. Society of Offshore and Polar Eng. (ISOPE), Maui, HI, 2011.

[17] U. A. Korde, "Efficient primary energy conversion in irregular waves," Ocean Eng., vol. 26, pp. 625-651, 1999.

[18] F. Fusco and J. Ringwood, "Short-term wave forecasting for real-time control of wave energy converters," IEEE Trans. Sustain. Energy, vol. 1, no. 2, pp. 99-106, Jul. 2010.

[19] K. Budal and J. Falnes, "The Norwegian wave-power buoy project," in Second Int. Symp. Wave Energy Utillization, Trondheim, Jun. 1982, pp. 323-344.
[20] J. Tedd and P. Frigaard, "Short term wave forecasting, using digita filters, for improved control of wave energy converters," in Proc. Int Offshore and Polar Eng. Conf. (ISOPE), 2007, pp. 388-394.

[21] M. Belmont, J. Horwood, R. Thurley, and J. Baker, "Filters for linear sea-wave prediction," Ocean Eng., vol. 33, pp. 2332-2351, 2006.

[22] H. Van Den Boom, OWME Project Makes Technological Breakthrough 2009, Marin Report.

[23] U. A. Korde, "On control approaches for efficient primary energy conversion in irregular waves," in Proc. IEEE Oceanic Eng. Society (OCEANS'98), 1998, vol. 3, pp. 1427-1431.

[24] U. A. Korde, "Control system applications in wave energy conversion," in Proc. OCEANS 2000 MTS/IEEE Conf. Exhibition, 2000, vol. 3, pp. $1817-1824$

[25] A. Babarit, G. Duclos, and A. Clement, "Comparison of latching control strategies for a heaving wave energy device in random sea," Appl. Ocean Res., vol. 26, pp. 227-238, 2004.

[26] F. Fusco, J.-C. Gilloteaux, and J. Ringwood, "A study on prediction requirements in time-domain control of wave energy converters," in Proc. Control Appl. Marine Syst. (CAMS), Germany, 2010, pp. 405-410.

[27] J. Cruz, Ocean Wave Energy. Current Status and Future Perspectives. New York: Springer-Verlag, 2008.

[28] F. Fusco and J. Ringwood, "A model for the sensitivity of non-causa control of wave energy converters to wave excitation force prediction errors," in Proc. 9th Eur. Wave and Tidal Energy Conf. (EWTEC), Southampton, U.K., 2011.

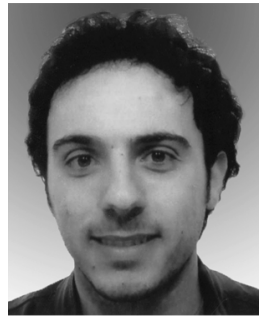

Francesco Fusco received the Master's degree in industrial automation engineering, in 2008, from Universita' Politecnica delle Marche (UNIVPM), Ancona, Italy, with his final project carried out at the National University of Ireland Maynooth (NUIM) Ireland. Since January 2009, he has been working toward the Ph.D. degree at the Center for Ocean Energy Research (COER), NUIM.

His research project is focused on the optimization of wave energy converters control systems through prediction of the future incident wave elevation or wave excitation force. In 2010 and 2011, he also worked as an occasional lecturer in the Electronic Engineering Department at NUIM.

Mr. Fusco is a Chartered Engineer in Italy.

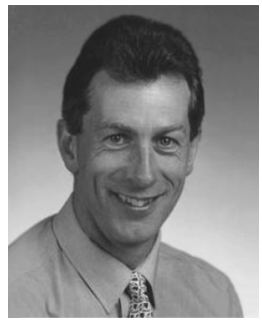

John V. Ringwood received the Diploma in electrical engineering from Dublin Institute of Technology and the Ph.D. degree (in control systems) from Strathclyde University, Scotland, in 1981 and 1985 , respectively.

$\mathrm{He}$ is currently Professor of Electronic Engineering with the National University of Ireland (NUI), Maynooth and Associate Dean for Engineering in the Faculty of Science and Engineering. $\mathrm{He}$ was Head of the Electronic Engineering Department at NUI Maynooth from 2000 until 2005, developing the Department from a greenfield site. His research interest cover time series modeling, wave energy, control of plasma processes, and biomedical engineering.

Dr. Ringwood is a Chartered Engineer and a Fellow of the Institution of Engineers of Ireland. 